\title{
Função Manual na Síndrome de Down
}

\author{
Manual Function in Down Syndrome
}

\author{
Función manual en el síndrome de Down
}

\author{
Aline Bernardes de Souza1 ${ }^{1}$, Silvana Maria Blascovi-Assis² \\ 1.Fisioterapeuta, Doutora em Distúrbios do Desenvolvimento, Centro Universitário de Brusque, Brusque- \\ SC, Brasil. \\ 2.Fisioterapeuta, Doutora em Educação Física, Universidade Presbiteriana Mackenzie, São Paulo-SP, Brasil.
}

\section{Resumo}

Introdução. A função manual envolve diferentes habilidades como força, destreza, precisão e controle de movimentos que tendem a interferir na rotina diária dos indivíduos. A força de preensão palmar vem sendo avaliada, pela literatura científica, como possibilidade única de quantificação da função manual dos indivíduos. Porém, pessoas com síndrome de Down (SD) apresentam alterações no desenvolvimento, as quais tendem a promover dados distintos de suas habilidades manuais o que dificultaria sua avaliação mediante uma única variável. Objetivo. Avaliar a força de preensão palmar, o desempenho funcional manual e a destreza manual em indivíduos com SD, comparando-os com um grupo controle e, investigar a possibilidade de utilização da força de preensão palmar como variável única na verificação da função manual na síndrome de Down. Método. Contou-se com a participação de 30 indivíduos com SD e 30 sem alteração no desenvolvimento, com idades entre 11 e 14 anos que foram avaliados pelo dinamômetro Jamar ${ }^{\circledR}$, Teste da Função manual de Jebsen-Taylor e Teste de Caixa e Blocos. Resultados. Notou-se em todos os testes um desempenho inferior do grupo com SD quando comparados ao grupo controle, sem diferenciação da performance entre a mão preferida e a mão não preferida, havendo uma correlação positiva entre as variáveis força de preensão palmar e tarefa de empilhar damas do teste de Jebsen-Taylor. Conclusão. Os indivíduos com síndrome de Down apresentam um desempenho manual inferior à população sadia. A utilização da Força de Preensão Palmar como única variável para mensuração da função manual na SD precisa ser melhor estudada.

Unitermos. Síndrome de Down; força da mão; destreza motora; lateralidade funcional

\begin{abstract}
Introduction. The manual function involves different skills such as strength, dexterity, precision and movement control that tend to interfere in the individuals' daily routine. The handgrip strength has been evaluated in the scientific literature as a unique possibility to quantify the manual function of individuals. However, people with Down syndrome (DS) have developmental changes, which tend to promote different data from their manual skills, which would make it difficult to assess using a single variable. Objective. To evaluate handgrip strength, manual functional performance and manual dexterity in individuals with DS, comparing them with a control group, and investigate the possibility of using handgrip strength as a single variable in the verification of hand function in Down syndrome. Method. Thirty individuals with DS and 30 without developmental change, aged from 11 to 14 years old, who were evaluated by the Jamar $\circledR$ dynamometer, the Jebsen-Taylor Manual Function Test and the Box and Blocks Test, participated in this study. Results. It was noted in all tests an inferior performance of the DS group when compared to the control group, with no differentiation in performance between the preferred hand and the non-preferred hand, with a positive correlation between the variables handgrip strength and task of stack checkers of the JebsenTaylor test. Conclusion. Individuals with Down syndrome have a lower manual performance than the healthy population. The use of handgrip strength as the only variable for measuring manual function in DS needs to be better studied.
\end{abstract}

Keywords. Down syndrome; hand strength; motor skills; functional laterality 


\section{Resumen}

Introducción. La función manual involucra diferentes habilidades como fuerza, destreza, precisión y control del movimiento que tienden a interferir en la rutina diaria de los individuos. La fuerza de la empuñadura ha sido evaluada en la literatura científica como una posibilidad única para cuantificar la función manual de los individuos. Sin embargo, las personas con síndrome de Down (SD) tienen cambios de desarrollo, que tienden a promover datos diferentes de sus habilidades manuales, lo que dificultaría la evaluación utilizando una sola variable. Objetivo. Evaluar la fuerza del agarre, el desempeño funcional manual y la destreza manual en personas con SD, comparándolos con un grupo de control e, investigar la posibilidad de utilizar la fuerza del agarre como una variable única en la verificación de la función de la mano en el síndrome de Down. Método. Participaron de este estudio treinta individuos con SD y 30 sin câmbios em el desarrollo, de entre 11 y 14 años, que fueron evaluados mediante el dinamómetro Jamar ${ }^{\circledR}$, el Test de Función Manual Jebsen-Taylor y el Test de Caja y Bloques. Resultados. Se observó en todas las pruebas un desempeño inferior del grupo DS en comparación con el grupo control, sin diferenciación en el desempeño entre la mano preferida y la mano no preferida, con una correlación positiva entre las variables fuerza de agarre y tarea de fichas de pila de la prueba de Jebsen-Taylor. Conclusión. los individuos con síndrome de Down tienen un rendimiento manual menor que la población sana. Es necesario estudiar mejor el uso de la fuerza de la empuñadura como única variable para medir la función manual en el síndrome de Down.

Palabras clave. Sindrome de Down; fuerza de la mano; habilidades motoras; lateralidad funcional

Trabalho realizado na Universidade Presbiteriana Mackenzie, São Paulo-SP, Brasil.

Conflito de interesse: não

Recebido em: 22/08/2021

Aceito em: 12/11/2021

Endereço para correspondência: Aline Bernardes de Souza. R. Dorval Luz 123. Santa Terezinha. Brusque-SC, Brasil. E-mail: alineberanrdessouza@yahoo.com.br

\section{INTRODUÇÃO}

A mão humana tem um importante papel na interação do indivíduo com 0 ambiente e no modo de vida independente. Entre as suas principais funções destacam-se a manipulação de objetos; a coordenação motora; o controle de forças e a precisão nas tarefas desempenhadas, como a escrita ${ }^{1-5}$.

O processo de desenvolvimento das habilidades manuais pode ser prejudicado pelas alterações sensoriais e tônicas do indivíduo, pelo déficit na coordenação e pelo pequeno tamanho de suas mãos e $\operatorname{dedos}^{1}$. 
Pessoas com síndrome de Down (SD), por exemplo, tendem a ter um pequeno tamanho de mãos associado a um quadro de hipotonia que promove um atraso no desenvolvimento de padrões motores manipulativos e um prejuízo no desempenho em suas atividades de vida diária68.

A função manual pode ser mensurada de diversas formas e de acordo com a habilidade a ser averiguada. A Força de Preensão Palmar (FPP) corresponde a habilidade mais avaliada na prática clínica e acadêmica ${ }^{5}$ que consiste na aferição da força de preensão máxima ao apertar, com uma das mãos, as duas hastes de um dinamômetro, em um único momento ${ }^{1}$.

Outro componente que vem sendo vastamente investigado pela comunidade científica é a destreza manual ${ }^{6-}$ 8. Esta corresponde ao desempenho motor e funcional, habilidoso e orientado, da mão e do braço durante a manipulação de um objeto ${ }^{9}$.

A destreza manual quando avaliada pelo Teste de Caixas e Blocos (TCB) na população brasileira com SD em idade escolar, vem demonstrando uma divergência entre os resultados dos estudos. Alguns indivíduos quando avaliados tenderam a apresentar uma constância no desempenho entre as faixas etárias e outros uma significativa evolução com o passar dos anos, sempre demonstrando um desempenho inferior ao seu grupo controle. A FPP também foi aferida nestes estudos demonstrando que o grupo com SD apresenta um desempenho inferior ao grupo controle 
com uma significativa evolução ao longo dos anos. A FPP e TCB não demonstraram uma correlação significativa entre si nestes estudos. Este fato demonstra que o entendimento prévio de que a FPP poderia ser uma referência para a avaliação da função manual não condiz para esta população estudada $8,10,11$.

Foi avaliada a relação entre a FPP, o desempenho funcional manual pelo Teste da Função Manual de JebsenTaylor (TFMJT) e a destreza manual pelo teste de Nove Pinos nos Buracos (9-PnB), em indivíduos adultos sadios ${ }^{5}$. Ao final do estudo, os autores constataram a presença de uma correlação moderada entre a FPP e o TFMJT e uma correlação fraca entre o TFMJT e o 9-PnB. Assim, os autores concluíram que ao se utilizar os dados da FPP como indicativo geral da função manual deve-se ter precaução na interpretação dos resultados, averiguando se esta variável contempla a coordenação e o controle do movimento.

Sendo assim, a função manual na SD precisa ser melhor investigada verificando o comportamento de suas variáveis manuais e a possibilidade do uso da FPP como um preditor de sua quantificação. Portanto, este estudo buscou avaliar a FPP e o desempenho funcional manual pelo TFMJT e a destreza manual pelo TCB, da mão preferida (MP) e não preferida (MNP) em indivíduos com SD e compará-los com um grupo controle além de verificar a possibilidade de utilização da FPP como variável única na verificação da função manual na síndrome de Down. 


\section{MÉTODO}

\section{Amostra}

Participaram deste estudo 30 crianças e jovens sem alteração no desenvolvimento que compuseram o grupo controle (GC) e 30 indivíduos com síndrome de Down (SD) que formaram o grupo com SD (GSD), de ambos os sexos, na faixa etária escolar de 11 a 14 anos.

Os participantes eram oriundos de instituições de ensino público regular e/ou instituições de atendimento especializado à pessoa com SD de cidades do interior do estado de São Paulo, Brasil. Indivíduos que apresentassem alterações ortopédicas e/ou neurológicas que pudessem interferir na realização dos testes foram excluídos.

Os procedimentos éticos seguiram os preceitos da Declaração de Helsinque e do Código de Nuremberg, respeitando as normas de pesquisa com Seres Humanos do Conselho Nacional de Saúde (Resolução CNS 466/12). A todos os pais ou responsáveis dos participantes bem como aos participantes foi entregue o Termo de Consentimento Livre e Esclarecido que foi assinado antes do início da coleta de dados. O presente estudo foi aprovado pelo Comitê de Ética em Pesquisa sob o parecer 343.289/CAAE 11719212.5.0000.0084.

\section{Procedimentos}

A preferência manual (PM) foi avaliada pelo protocolo de Van Strien de 2002, versão curta ${ }^{12}$, que se compõe da simulação de dez atividades manuais pelo participante. 
Verificou-se qual a mão utilizada para realizar cada tarefa anotando-a no protocolo. Posteriormente, averiguou-se qual foi a mão mais utilizada nas tarefas e definiu-se a preferência manual do indivíduo. A análise da consistência da PM não foi utilizada para este estudo.

Para a avaliação da FPP contou-se com o auxílio do dinamômetro Jamar ${ }^{\circledR}$ que apresenta um sistema hidráulico de aferição de tensão, de uso simples, no qual o sujeito aperta as barras do instrumento, ocasionando uma alteração na resistência dos aferidores que é diretamente proporcional à força exercida pelas mãos. Para tal, o participante permaneceu sentado, com os pés apoiados no chão, joelho e quadril flexionados a 90 graus. A barra do dinamômetro foi ajustada na posição 2. O dinamômetro foi sustentado pelo avaliador durante a execução do teste.

Para a análise do desempenho funcional manual foi utilizado o teste de função manual de Jebsen-Taylor que avalia o uso funcional da mão, documentando sua habilidade em cada categoria analisada: escrita, virar cartas (VC), pegar pequenos objetos (PPO), simulação de alimentação (SA), empilhar damas (ED), movimentar objetos leves (MOL) e pesados (MOP). Os escores consistem na mensuração do tempo, em segundos, utilizado em cada atividade ${ }^{13}$. O procedimento foi iniciado com a mão não preferida (MNP) do indivíduo e posteriormente executado com a mão preferida (MP), seguindo as recomendações originais do teste ${ }^{14}$.

Foi utilizado também a avaliação da destreza manual com o uso do Teste de Caixa e Blocos (TCB) desenvolvido 
por Mathiowetz et al. ${ }^{15}$, que avalia a habilidade e a destreza dos indivíduos em levar a maior quantidade de cubos presente num compartimento da caixa para a outra repartição, um de cada vez, não soltando o cubo até seus dedos encostarem-se à caixa, por um minuto.

Todos os procedimentos foram realizados por três vezes com cada mão, alternando a execução entre elas, sendo considerado o melhor resultado em cada mão para análise de dados.

\section{Análise Estatística}

Para análise estatística dos dados foi utilizado o pacote estatístico SPSS - Statistical Package for Social Sciences para o Windows, versão 1.8 , que proporciona o cálculo e as informações estatísticas ${ }^{16}$.

Como os dados deste estudo não apresentaram uma distribuição normal pelo teste de Kolmogorov-Smirnov, utilizou-se, então, a estatística não-paramétrica para análise de todos os dados.

Assim, para a análise pareada dos dados utilizou-se o teste de Wilcoxon e para correlação entre as médias das o teste de Spearman. Para ambas as análises se utilizou um nível de significância igual a 5\%.

Por não ser possível equiparar o número da amostra por faixa etária e sexo entre os grupos utilizou-se a média de cada grupo na análise estatística. 


\section{RESULTADOS}

A avaliação da PM tanto para o GC como para o GSD demonstrou os resultados apresentados na Tabela 1. Notase que a maioria dos indivíduos, em ambos os grupos, teve uma PM à direita.

Tabela 1. Porcentagem de preferência manual nos grupos aferida pela versão curta do protocolo de Van Strien (2002) ${ }^{12}$.

\begin{tabular}{ccc}
\hline $\begin{array}{c}\text { GRUPO/ } \\
\text { PM }\end{array}$ & $\begin{array}{c}\text { GC } \\
(\mathbf{n = 3 0})\end{array}$ & $\begin{array}{c}\text { GSD } \\
(\mathbf{n = 3 0})\end{array}$ \\
\hline DIREITA & 86,7 & 60 \\
ESQUERDA & 13,3 & 33,3 \\
AMBIDESTRA & 0,0 & 6,7 \\
\hline
\end{tabular}

Para efeito de enquadramento e comparação entre a mão preferida (MP) e a mão não preferida (MNP) a PM ambidestra do GSD foi considerada direita, para este estudo, por ser a predominância do grupo.

A força de preensão palmar (FPP) na MP foi maior para dos indivíduos pertencentes ao GC do que para MNP $(23,83 \pm 6,33 \mathrm{kgf} ; \quad 21,16 \pm 6,02 \mathrm{~kg}$; respectivamente; $p=0,001)$.

Já O GSD apresentou média de FPP na MP de $13,38 \pm 5,60 \mathrm{kgf}$ e valor médio de FPP na MNP de $12,93 \pm 6,33 \mathrm{kgf}$, sem diferença entre as variáveis envolvidas $(p=0,225)$.

A destreza manual avaliada pelo Teste de Caixa e Blocos (TCB) no GC apresentou maior média $(75,33 \pm 12,78)$ de 
blocos transferidos por minuto pela MP e do que pela MNP $(73,76 \pm 9,35 ; p=0,014)$. Já para o GSD não houve diferença significante, foram em média 24,66 \$9,39 blocos deslocados

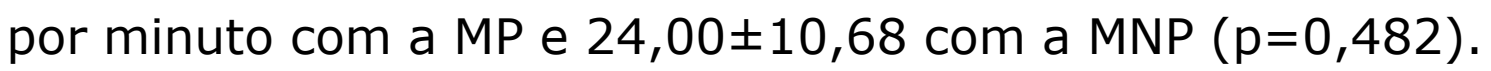

As tarefas relacionadas ao teste de função manual de Jebsen-Taylor para o GC e para o GSD mostraram os tempos médios (segundos) apresentados na Tabela 2. O teste de escrita foi desconsiderado nesse estudo uma vez que o GSD não conseguiu cumprir a tarefa.

Tabela 2. Tempo médio em segundos para as tarefas do teste Jebsen-Taylor de acordo com a PM.

\begin{tabular}{lcccccccc}
\hline TAREFA/ PM & MP & DP & MNP & DP & MP & DP & MNP & DP \\
& GC & & GC & & GSD & & GSD & \\
\hline VC & 4,76 & 1,20 & 4,77 & 1,01 & 13,13 & 5,85 & 13,37 & 6,46 \\
PPO & 5,82 & 0,79 & 5,83 & 0,72 & 13,63 & 4,15 & 13,41 & 3,73 \\
ED & 1,34 & 0,32 & 1,45 & 0,35 & 4,19 & 1,53 & 4,66 & 1,88 \\
AS & 10,21 & 1,86 & 11,87 & 1,90 & 19,37 & 9,33 & 24,57 & 15,12 \\
MOL & 3,53 & 0,42 & 3,72 & 0,37 & 7,76 & 2,20 & 7,87 & 2,21 \\
MOP & 3,97 & 0,54 & 4,07 & 0,61 & 9,29 & 3,50 & 10,38 & 3,58 \\
\hline
\end{tabular}

Houve diferença entre a MP e a MNP nas tarefas de Jebsen-Taylor, para o GC para a simulação da alimentação $(p=0,01)$ e para a movimentação de objetos leves $(p=0,030)$. Nas demais tarefas não foram significantes: virar cartas $(p=0,762)$, pegar pequenos objetos $(p=0,762)$, empilhar damas $(p=0,54)$ e movimentar objetos pesados $(p=0,173)$. Para o GSD houve diferença significante entre a MP e a MNP na tarefa de empilhar damas $(p=0,016)$, simular 
alimentação $(p=0,018)$ e movimentar objetos pesados $(p=0,011)$. As demais tarefas não apresentaram diferenças significantes: virar cartas $(p=0,517)$, pegar pequenos objetos $(p=0,510)$ e movimentar objetos leves $(p=0,918)$.

A comparação entre o GC e o GSD demonstrou a existência de uma diferença entre os valores dos grupos, tanto na MP como na MNP, em todas as variáveis averiguadas $(p=0,000)$.

Não houve correlação entre as variáveis FPP e TCB, no GC: $M P(p=0,849$, rho=0,36) e MNP $(p=0,556$, rho=0,112). O mesmo resultado encontrou-se para o GSD: FPPXTCB com MP: $p=0,881$, rho $=0,028$ e FPPxTCB com MNP: $p=0,140$, rho $=0,276$.

Houve correlação entre as seis tarefas realizadas pela MP e a FPP no GC, para FPPxMOP: $p=0,026$, rho=0,407. Para a MNP, houve, somente, uma correlação negativa para FPPXED: $p=0,050$, rho $=-0,361$.

Para o GSD houve correlação negativa nas aferições com a MP com as seis tarefas do TFMJT entre FPPxED: $p=0,011$, rho $=-0,456$. Para as aferições com a MNP não houve correlações significantes.

\section{DISCUSSÃO}

Diante dos resultados apresentados podemos observar que o GSD teve maior número de indivíduos com preferência manual à esquerda (33,3\%) quando comparado com GC $(13,3 \%)$. A presença de indivíduos ambidestros, sem 
preferência lateral definida, também foi notada em $6,7 \%$ do GSD.

Em todas as avaliações realizadas notou-se que as médias dos resultados apresentados pelo GSD foram inferiores ao GC. Esse fato pode estar relacionado às características típicas da própria síndrome como a hipotonia muscular, o atraso no desenvolvimento neuro motor e na motricidade global e fina, a baixa eficiência na manipulação de objetos, o pequeno tamanho das mãos, a hipermobilidade articular, o déficit intelectual, a dificuldade de atenção, concentração na realização das tarefas e no processamento de informações como vem sendo observado em estudos anteriores $8,10,17,18$.

Os resultados inferiores nos testes de função manual pelo GSD demonstram também as dificuldades no controle e planejamento motor, na percepção ocular e na integração visuo motora que estes indivíduos apresentam ${ }^{18}$.

De maneira geral, os dois grupos apresentaram melhores resultados, em todos os testes, com a MP do que com a MNP, embora nem sempre significante. Este fato relaciona-se aos fundamentos da lateralidade que supõem que as atividades sejam realizadas com uma força, exatidão, preferência, destreza e coordenação superior com a MP quando comparada com a MNP, sendo estes fatores fundamentais para a aprendizagem escolar ${ }^{19}$.

O GSD, diferentemente do $G C$, não apresentou diferenças na FPP e no TCB entre a MP e a MNP demonstrando, assim, uma possível tendência a simetria 
motora e um déficit na especialização unilateral do corpo. A hipotonia generalizada somada à dificuldade da consistência da dominância lateral pode estar relacionada a este dado.

Diferenças na atuação da mão dominante versus mão não dominante em indivíduos com SD brasileiros também não foram observadas em estudo que avaliou 50 sujeitos com SD de 7 a 9 anos e de 14 a 15 anos avaliados com 0 $\mathrm{TCB}^{11}$.

Em outro estudo, o GSD brasileiro também não apresentou correlação estatística entre as variáveis FPP e TCB em ambas as mãos ${ }^{10}$. Todavia, os mesmos autores encontraram esta correlação para o GC por eles estudado, o que não ocorreu no presente estudo. Tal fato merece ser mais bem explorado em estudos futuros.

A prática do protocolo proposto por Jebsen-Taylor para avaliar o desempenho funcional manual nos indivíduos com SD mostrou-se sensível a estes uma vez que eles conseguiram executar as tarefas sugeridas pelo autor com exceção da primeira atividade que se refere à escrita.

Durante a prática da tarefa de escrita, tentou-se, inicialmente, que os sujeitos com SD redigissem uma frase simples com 24 letras, da mesma forma que o GC, mas eles não conseguiram. Experimentou-se, então, a descrição do seu nome em uma folha em branco. Esta, também não foi possível para todos os avaliados. Decidiu-se por fim eliminar esta tarefa do conjunto de avaliações.

Essa dificuldade pode estar relacionada às características peculiares da SD, como o déficit intelectual, 
porém outras variáveis podem interferir de modo associado como a consistência da lateralização como já observado em outros estudos ${ }^{20,21}$ nos quais os escolares mostram que a preferência lateral mal definida apresenta uma relação significativa com as funções corticais e a alfabetização.

Estudo onde se aplicou o protocolo de Jebsen-Taylor em um grupo de 36 adultos jovens sadios, optou-se pela eliminação da prova de escrita considerando que as frases propostas originalmente estavam em inglês ${ }^{5}$. Porém uma adaptação desta tarefa poderá ser futuramente proposta e estudada para sua aplicação às pessoas com SD.

As demais ações foram executadas pelo GSD de maneira mais lenta do que o GC como visualizado nos resultados apresentados. Pelo teste apresentar seis tarefas, com exceção da escrita, estes se apresentavam cansados e desestimulados ao realizar o teste com a MP e a MNP por três vezes, sendo necessário, muitas vezes, insistir para que completassem todo o protocolo proposto para esse estudo.

Durante a tarefa de virar cartas muitos participantes do GSD tentavam utilizar a mão contralateral a que estava executando o teste para virar a próxima carta.

Ao longo da tarefa de pegar pequenos objetos alguns demonstraram dificuldades em retirar o objeto de cima da superfície, agarrando-os com o movimento de pinça atípica. $\mathrm{O}$ ato motor em pinça atípica nestes indivíduos já foi observado $^{18} \mathrm{em}$ estudo que examinou o controle motor destes em tarefas de destreza manual. 
$\mathrm{Na}$ simulação da alimentação estes possuíam dificuldade em conseguir encaixar a colher embaixo dos feijões e, nas tarefas de movimentar objetos leves e pesados muitos não conseguiam transportá-los ordenadamente sendo, às vezes, necessária a orientação verbal e manual do avaliador para a realização correta do procedimento. A limitação em realizar habilidades motoras a partir de uma instrução verbal já foi mencionada pela literatura ao avaliar a aquisição da habilidade motora a partir de instruções verbais e motoras ${ }^{22}$.

Nas tarefas propostas pelo teste Jebsen-Taylor ocorreram diferenças estatísticas entre as MP e MNP nos dois grupos, sendo no GC nas atividades de simular alimentação e movimentar objetos leves e, no GSD, nas ações de empilhar damas, simular alimentação e movimentar objetos pesados. A disparidade na simulação da alimentação justifica-se, talvez, por ser essa uma tarefa corriqueira e que exige o uso e a especialização de um membro ao ser realizada com uma colher não sendo necessária realizá-la com o auxílio do membro contralateral.

A tarefa de empilhar blocos e construir um traço na vertical já foi verificada em indivíduos com SD de idade escolar. Os investigadores observaram que os indivíduos com SD apresentavam desempenho inferior quando comparados com o grupo controle e, relacionaram esta atividade à coordenação olho mão e ao controle dos dedos sendo a base para o desenvolvimento das habilidades motoras finas complexas ${ }^{23}$. 
Diferentemente do que se esperava a correlação entre FPP e MOP, no GC, se mostrou positiva demonstrando que quanto maior FPP, maior o tempo de execução da tarefa de MOP. Já a correlação entre FPP e ED se apresentou negativa tanto para o GC para MP e para o GSD para a MNP demonstrando que quanto maior a FPP menor o tempo de execução da tarefa de ED.

A partir desses achados pode-se considerar que existem diferenças nas habilidades manuais entre os grupos, com desvantagem para GSD, com maior evidência de desempenho entre MP e MNP para o GC. Constatou-se ainda que tanto o TCB como o teste de Jebsen Taylor podem ser utilizados para a população com SD, com exceção da tarefa de escrita e, que a utilização da FPP como única variável para mensuração da função manual na SD precisa ser melhor estudada.

A tarefa de escrita, do teste de Jebsen-Taylor, merece ser melhor investigado na população com SD ampliando o conhecimento das habilidades manuais nestes e suas correlações.

Pesquisas que envolvam uma amostra com equiparação de faixas etárias entre grupos, sexo e PM devem ser, também, estimulados e realizados para elucidarem melhor estas temáticas.

\section{CONCLUSÃO}

Os indivíduos com SD aqui avaliados apresentaram um desempenho manual médio inferior ao GC. As tarefas de FPP 
e TCB não demonstraram diferenças na atuação entre MP e MNP no grupo com SD.

A correlação entre a FPP e TCB, no GSD, não foi observada neste estudo, demonstrando, mais uma vez, que a força não interfere no desempenho da destreza manual dos indivíduos com SD quando avaliados por estes testes. Já a correlação entre a FPP e as tarefas de Jebsen-Taylor ocorreu apenas para a tarefa de ED para a MNP.

Sendo assim, a utilização da FPP como única variável para mensuração da função manual na SD precisa ser melhor estudada.

\section{AGRADECIMENTOS}

À Coordenação de Aperfeiçoamento de Pessoal de Nível Superior - Capes.

\section{REFERÊNCIAS}

1. Esteves AC, Reis DC, Caldeira RM, Leite RM, Moro ARP, JR NGB. Força de preensão, lateralidade, sexo e características antropométricas da mão de crianças em idade escolar. Rev Bras Cineantropom Desemp Hum 2005;7:69-75.

https://periodicos.ufsc.br/index.php/rbcdh/article/view/3799/16773

2.Jones LA, Lederman SJ. Book human hand function. New York: Oxford University Press; 2006. http://dx.doi.org/10.1093/acpro f:oso/9780195173154.001.0001

3. Freitas $\mathrm{PB}$, Krishnan $\mathrm{V}$, Jaric $\mathrm{S}$. Force coordination in object manipulation. J Hum Kinet 2008;20:37-51.

http://dx.doi.org/10.2478/v10078-008-0016-8

4.Zatsiorsky VM, Latash ML. MultiBinger prehension: an overview. J Mot Behav 2008;40:446-76.

http://dx.doi.org/10.3200/JMBR.40.5.446-476

5. Lima KCA, Francisco MM, Freitas PB. Relação entre os desempenhos em diferentes testes frequentemente utilizados na avaliação da função manual. Fisiot Mov 2012;25:517-24.

http://dx.doi.org/10.1590/S0103-51502012000300007

6.Guimarães R, Blascovi-Assis SM. Uso do teste caixa e blocos na avaliação de destreza manual em crianças e jovens com síndrome de 
Down. Rev Ter Ocup Univ São Paulo 2012;23:98-106. https://doi.org/10.11606/issn.2238-6149.v23i1p98-106

7.Rufino LA, Blascovi-Assis SM, Souza AB, Verginassi G, Cymrot R. Avaliação da destreza manual em pessoas com síndrome de Down: comparação entre teste de caixa e blocos, Minessota e Jebsen-Taylor. Fisiot Bras 2016;17:188-96. http://dx.doi.org/10.33233/fb.v17i3.476 8.Souza AB, Cymrot R, Vianna DL, Caromano FA, Blascovi-Assis SM. Antropometria da mão e função manual de crianças e jovens com síndrome de Down. Rev Port Ciênc Desp 2013;13:78-89. http://dx.doi.org/10.5628/rpcd.13.03.78

9. Magill RA. Aprendizagem motora: conceitos e aplicações. São Paulo: Edgard Blucher; 2000.

10.Priosti PA, Blascovi-Assis SM, Cymrot R, Vianna D, Caromano F. Força de Preensão e Destreza Manual na criança com síndrome de Down. Fisiot Pesq 2013;20:278-85. http://dx.doi.org/10.1590/S180929502013000300013

11.Guimarães R, Blascovi-Assis SM, Macedo EC. Efeito da dominância lateral no desempenho da destreza manual em pessoas com síndrome de Down. Acta Fisiátr 2012;19:6-10. http://dx.doi.org/10.5935/01047795.20120002

12.Van Strien J. The Dutch Handedness Questionnaire. FSW, Department of Psychology. Rotterdam: Erasmus University Rotterdam; 2002.

13.Rodrigues AMVN, Mancini MC, Vaz D, Silva LC. Uso de órtese para abdução de polegar no desempenho funcional de criança portadora de paralisia cerebral: estudo de caso único. Rev Bras Saúde Mater Inf 2007;7:423-36.

http://dx.doi.org/10.1590/S1519-

$\underline{38292007000400010}$

14.Jebsen RH, Taylor N, Trieschmann RB, Trotter MJ, Howard LA. An Objective and Standardized test of Hand Function. Arch Phys Med Rehabil 1969;50:311-9. https://pubmed.ncbi.nlm.nih.gov/5788487/ 15. Mathiowetz V, Volland G, Kashman N, Weber K. Adult Norms for the Box and Block Test of Manual Dexterity. Am J Occupat Ther 1985;39:386-91. http://dx.doi.org/10.5014/ajot.39.6.386

16.Bisquerra R, Sarriera JC, Martínez F. Introdução à Estatístca: enfoque informático com o pacote estatístico SPSS. Porto Alegre: Artemed, 2004.

17.Souza A, Cymrot R, Vianna D, Caromano F, Blascovi-Assis SM. Síndrome de Down: correlação entre o desempenho funcional com a força de preensão palmar e a destreza manual. Fisiot Bras 2012;13:211-5. https://doi.org/10.33233/fb.v13i3.540

18. Jover M, Ayoun C, Berton C, Carlier M. Specific grasp characteristics of children with trisomy 21. Develop Psycobiol 2010;52:782-93. http://dx.doi.org/10.1002/dev.20474

19. Linares P. Educación psicomotriz e aprendizaje escolar: motricidade y disgrafia. Madrid: Polibea; 1993.

20.Guardiolla A, Ferreira LTC, Rotta NT. Associação entre desempenho das funções corticais e alfabetização em uma amostra de escolares de 
primeira série de Porto Alegre. Arq Neuropsiquiatr 1998;56:2818. https://doi.org/10.1590/S0004-282X1998000200019

21.Corballis MC, Hattie J, Fletcher R. Handedness and intellectual achievement: an even-handed look. Neuropsychol 2008;46:374-8. http://dx.doi.org/10.1016/j.neuropsychologia.2007.09.009

22. Meegan S, Maraj B, Weeks D, Chua R. Gross motor skill aquisition in adolescents with Down syndrome. Syndr Res Pract 2006;9:75-80. http://dx.doi.org/10.3104/reports.298

23.Coppede AC, Campos AC, Santos DCC, Rocha NACF. Desempenho motor fino e funcionalidade em crianças com síndrome de Down. Fisioter Pesqu 2012;19:363-8. http://dx.doi.org/10.1590/S1809$\underline{29502012000400012}$ 